\title{
openheart Anticoagulation trends in adults aged 65 years and over with atrial fibrillation: a cohort study
}

\author{
Jenny Lund (1D), Catherine L Saunders, Duncan Edwards, Jonathan Mant
}

\begin{abstract}
- Additional supplemental material is published online only. To view, please visit the journal online (http://dx.doi.org/10.
\end{abstract} 1136/openhrt-2021-001737)

To cite: Lund J, Saunders CL, Edwards D, et al. Anticoagulation trends in adults aged 65 years and over with atrial fibrillation: a cohort study. Open Heart 2021;8:e001737. doi:10.1136/ openhrt-2021-001737

Received 17 June 2021 Accepted 16 July 2021

\section{Check for updates}

(C) Author(s) (or their employer(s)) 2021. Re-use permitted under CC BY. Published by BMJ.

Primary Care Unit, Department of Public Health and Primary Care, University of Cambridge, Cambridge, UK

Correspondence to Dr Jenny Lund; j1897@medschl. cam.ac.uk

\section{ABSTRACT}

Objective To describe patterns of anticoagulation prescription and persistence for those aged $\geq 65$ years with atrial fibrillation (AF).

Methods Descriptive cohort study using electronic general practice records of patients in England, who attended an influenza vaccination aged $\geq 65$ years and were diagnosed with AF between 2008 and 2018. Patients were stratified by 10 -year age group and year of diagnosis. Proportion anticoagulated, type of anticoagulation (direct oral anticoagulant (DOAC) or warfarin) initiated at diagnosis and persistence with anticoagulation over time are reported.

Results 42290 patients (49\% female), aged 65-74 $(n=11722), 75-84(n=19055)$ and $85+(n=11513)$ years at AF diagnosis are included. Prescription of anticoagulation at diagnosis increased over the time period from $55 \%$ to $86 \%$ in people aged $65-74$ years, from $54 \%$ to $86 \%$ in people aged $75-84$ years and from $27 \%$ to $75 \%$ in people aged 85 years and over. By 2018 , $92 \%$ of patients with newly diagnosed AF were started on a DOAC. Survivor function for 5 -year persistence in patients prescribed DOAC was $0.80(95 \% \mathrm{Cl} 0.77$ to 0.82$)$ and for warfarin 0.71 (95\% $\mathrm{Cl} 0.70$ to 0.72$)$. Survivor function for any anticoagulation at 5 years was $0.79(95 \%$ $\mathrm{Cl} 0.78$ to 0.81 ), 0.73 (95\% Cl 0.72 to 0.75 ) and 0.58 (95\% $\mathrm{Cl} 0.59$ to 0.64 ) for people aged $65-74,75-84$ and $85+$ years, respectively.

Conclusions Rates of anticoagulation in AF in those aged $\geq 65$ years have increased from 2008 to 2018, over which time period there has been a shift from initiating anticoagulation with warfarin to DOAC. Persistence with anticoagulation is higher in people on DOACs than on warfarin and in people aged $<85$ years.

\section{INTRODUCTION}

Atrial fibrillation $(\mathrm{AF})$ is an independent risk factor for stroke. Incidence of AF increases from 6 per 1000 in those aged $60-69$ years to 39 per 1000 in those aged $80-89$ years. Thirty-one per cent of strokes in those aged 80-89 years may be attributed to $\mathrm{AF}$, compared with $7.3 \%$ in those aged $60-69 .{ }^{1}$

Oral anticoagulation reduces the risk of stroke by $65 \%$. Although use is increasing, it is still underused in the UK, with $22 \%$ of

\section{Key questions}

What is already known about this subject?

- Anticoagulation is a highly effective way of reducing the risk of stroke associated with atrial fibrillation (AF), but is underused, particularly in older people.

- The introduction of direct oral anticoagulant (DOAC) has been associated with increasing use of anticoagulation in $\mathrm{AF}$.

- The UK National Screening Committee has stated that insufficient evidence about anticoagulation prescribing patterns, compliance and persistence is one of the barriers to a national screening programme for AF.

What does this study add?

- Our study provides up-to-date information on anticoagulation for $\mathrm{AF}$ in older people who are most at risk of AF-related stroke and highlights particular increases in use of anticoagulation in people aged 85 years and over.

- DOACs are now the major class of anticoagulant prescribed to patients with new AF in UK general practice.

- Long-term persistence with anticoagulation is higher with D0ACs than warfarin, but drops in all age groups over 5 years.

How might this impact on clinical practice?

- Improved uptake of anticoagulation at all ages removes one of the potential barriers to screening for AF, but new strategies may be needed to enhance long-term persistence with treatment.

eligible patients not receiving treatment in 2018. ${ }^{23}$

Warfarin, a vitamin $\mathrm{K}$ antagonist, was the first oral anticoagulant available and was licenced in the USA in 1954. Dabigatran was the first of the direct oral anticoagulation (DOAC) medications licensed for use in the UK in 2008. This has been followed by others in this group, including rivaroxaban, apixaban and edoxaban. Their arrival has been associated with an increase in prescribing of anticoagulation in non-valvular AF for all age groups. $^{4}$ 
Since risk of stroke in AF rises with age, the potential benefit from anticoagulation also rises with age, although so do risk of bleeding complications. ${ }^{5}$ The National Institute for Health and Care Excellence guidance recommends the use of anticoagulation in all those with a $\mathrm{CHA}_{2} \mathrm{DS}_{2}$ VASC2 score of 2 or more and the consideration of anticoagulation in those with a score of 1 where this is not due to gender, which means all patients aged $\geq 65$ years with $\mathrm{AF}$ are potential candidates for anticoagulation, and all patients aged $\geq 75$ years should be offered such treatment. Historically, older people have been less likely to receive anticoagulation despite evidence that the potential benefit is greater than the risk. ${ }^{6}$

$\mathrm{AF}$ has been considered as a candidate for a national screening programme in the UK. One of the criteria for adopting screening is that the clinical management of the condition should be optimised prior to implementation of screening. ${ }^{7}$ The UK National Screening Committee reviewed patterns of anticoagulation prescribing for $\mathrm{AF}$ in 2019. It concluded that there was insufficient evidence of optimised compliance, and around prescribing patterns of anticoagulation in $\mathrm{AF}$, in part because of lack of evidence about anticoagulation persistence over time. ${ }^{8}$

The objective of this study is therefore to provide this evidence of patterns of anticoagulation prescribing and persistence by 10 -year age group over the age of 65 years.

We answered three research questions using an electronic primary care database study:
1. How has the proportion of people with AF aged over 65 years who are anticoagulated changed between 2008 and 2018?

2. How has the type of anticoagulant prescribed changed for people with incident AF?

3. How is anticoagulation persistence over the age of 65 years affected by age and type of anticoagulation?

\section{METHOD}

\section{Data and population}

Data were extracted from the Clinical Practice Research Datalink (CPRD), a primary care research database of anonymised, electronic coded records from the Vision computer system. ${ }^{9}$ All clinical activity (including diagnoses and prescribing) in primary care are recorded using clinical codes. The dataset on which these analyses were performed was originally extracted to explore the impact of case finding for $\mathrm{AF}$ at the time of influenza vaccination. Influenza vaccination is offered to everyone over the age of 65 years in the $\mathrm{UK}$, and uptake in this age group over the time period of the study was $71 \%-75 \% .^{10}$

Patients included in this study were registered with a general practitioner (GP) in England, had records which fulfilled CPRD data quality standards, ${ }^{9}$ had a first diagnosis of AF between 1 September 2008 and 31 August 2018 and attended at least one influenza vaccine after the age of 65 years and during the study period, with at least 1-year registration before their first eligible influenza

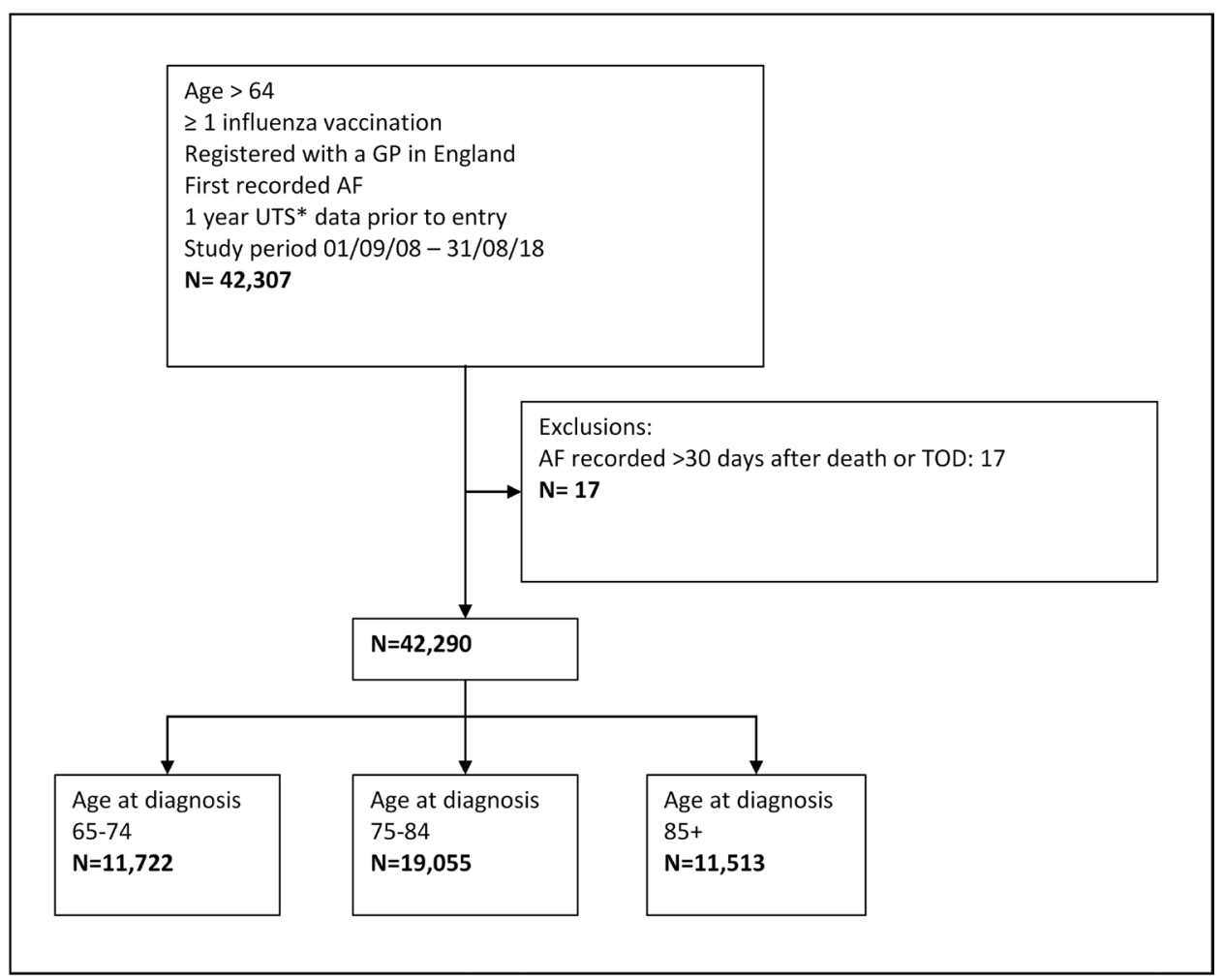

Figure 1 Patient flow diagram. AF, atrial fibrillation; CPRD, Clinical Practice Research Datalink; GP, general practitioner; UTS, up to standard: CPRD defined criteria for data quality; ${ }^{9} \mathrm{TOD}$, transfer out date :Date at which patients transfer to another practice. 

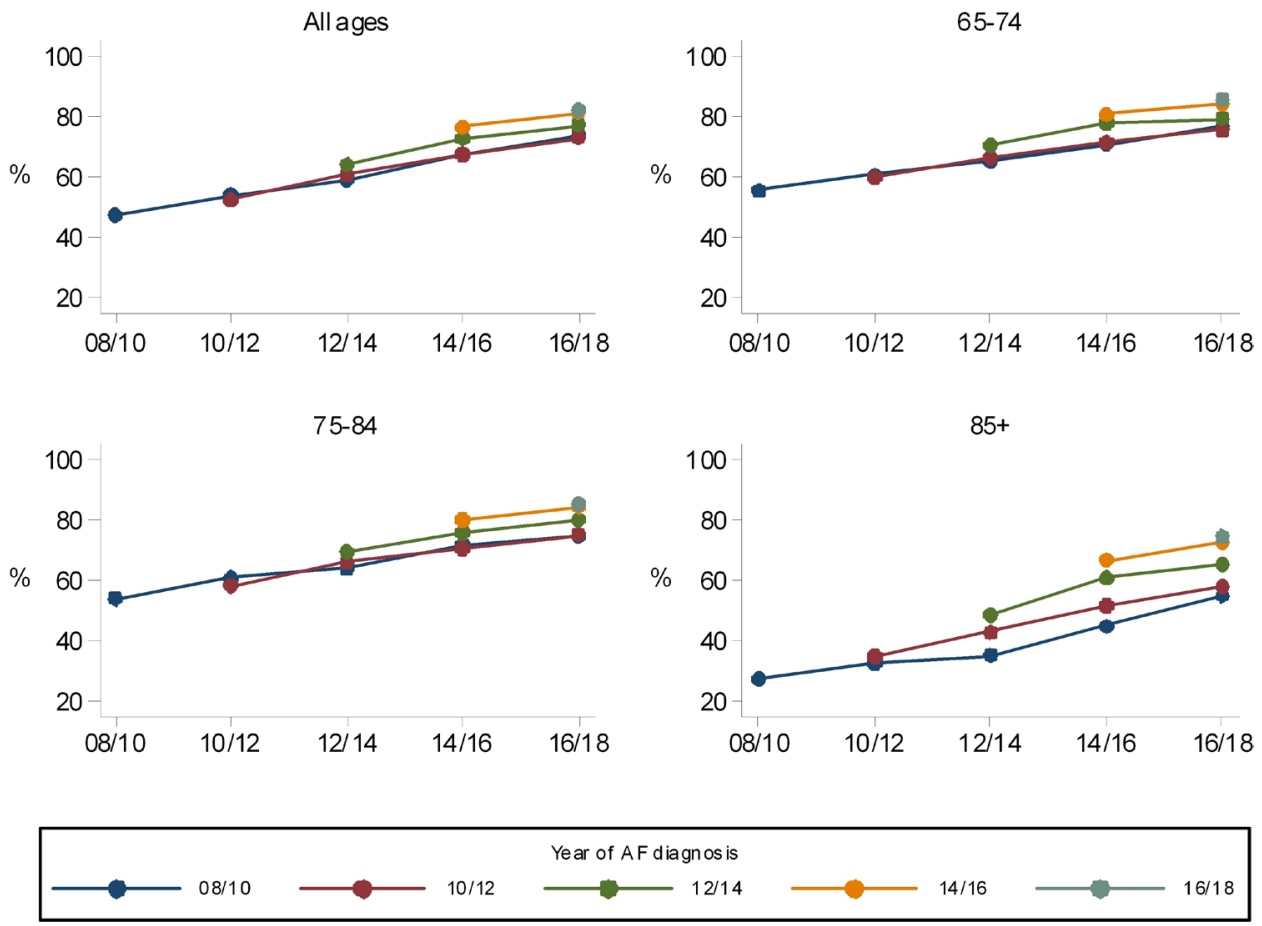

Figure 2 Anticoagulation for atrial fibrillation (AF) by cohort; age and year of diagnosis.

vaccination. For each patient, follow-up started at the date of first recorded AF diagnosis and ended when the patient left the GP practice or died, the practice stopped contributing data to CPRD, or 31 August 2018, whichever was earliest. Further limited exclusions for data quality were made; patients with an AF diagnosis recorded $>30$ days after the end of follow-up were excluded. Details of patient selection, inclusion and exclusion criteria are given in figure 1.

\section{Definitions of AF and anticoagulation}

We analysed cases of AF diagnosed each year from 1 September 2008 to 31 August 2018.

The date and type of first anticoagulation are defined at the first record of an oral anticoagulant prescription. For all analyses, we defined an anticoagulation prescription as a medication from the prespecified list (online supplemental appendix 1) with a tablet quantity greater than zero.

Lists of codes which record AF diagnoses and anticoagulation prescribing are available in online supplemental data appendix 1.

\section{Demographic and clinical characteristics}

We defined three age groups, 65-74, 75-84 and 85+ years. People aged 85 years and over were grouped because of small numbers and the risk of statistical disclosure. We described the clinical characteristics of the study population on the day before $\mathrm{AF}$ diagnosis as the count of long-term conditions (out of the 20 included in the Cambridge multimorbidity score). ${ }^{11}$ Deprivation was defined as the index of multiple deprivation (IMD) score of the GP practice attended by each cohort member, linked and provided by CPRD. IMD score is a small area measure of deprivation, measured across seven domains and reported for 32844 small areas in England. ${ }^{12}$

\section{Analyses}

In our first analysis, we explored changes in the proportion of people with AF prescribed anticoagulation by age and year. Each cohort was defined by age group at diagnosis and 2-year diagnosis interval. Patients were included in the denominator of each 2-year time point analysis if they had contributed data at any point during the 2-year time interval and were defined as anticoagulated if they had received any prescription of anticoagulation in this time.

For our second analysis, we included incident AF cases who received anticoagulation within 365 days of diagnosis and calculated the proportion, with $95 \%$ CIs, of these, stratified by age group, who received a DOAC as the first prescription after AF diagnosis. For this analysis, patients contributed to both the numerator and denominator of any year if they had a minimum of 365 days of follow-up after AF diagnosis.

In our final analyses, we described the time to end of anticoagulation use, stratified by age group at diagnosis and by age and type of anticoagulation. Analysis entry point was defined as the later of $\mathrm{AF}$ diagnosis date or first anticoagulation prescription date. The date of end of anticoagulation is defined as the date of the last anticoagulation prescription if this is $>90$ days before leaving the sample. If the patient left the sample for any reason within 90 days of anticoagulation prescription, they were censored at the leaving date. For this analysis, type of anticoagulation was defined as one of four categories; warfarin only, DOAC only, warfarin changed to 


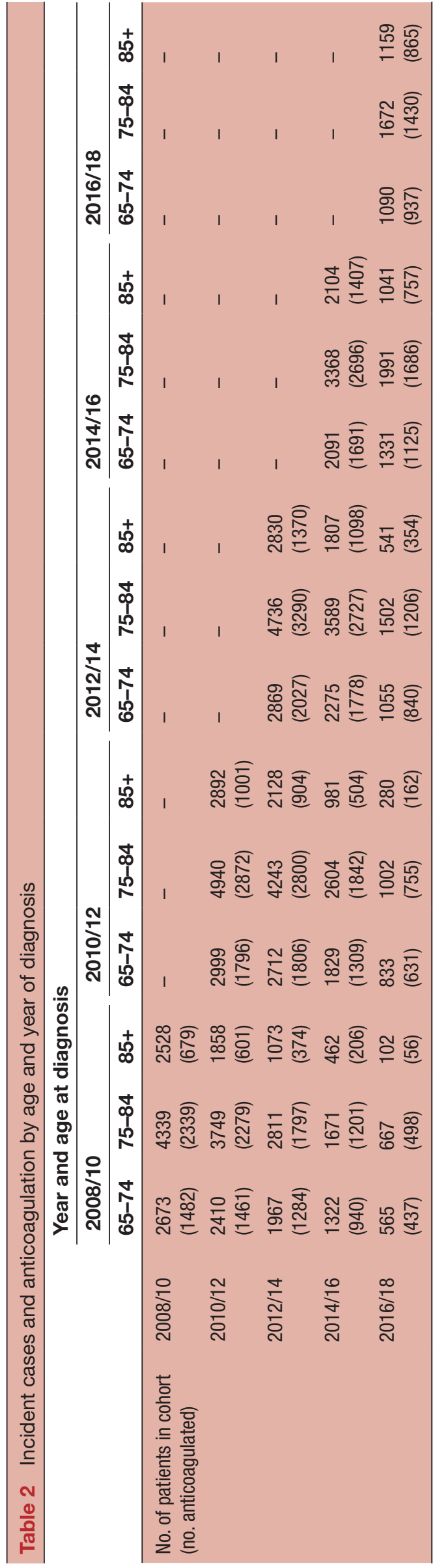

DOAC during follow-up and DOAC changed to warfarin. Patients with a prescription for a vitamin $\mathrm{K}$ antagonist other than warfarin were excluded due to small numbers. The survivor function was calculated, with $95 \%$ CIs, at the 5 -year time point for all age and medication cohorts.

All data analysis was completed using STATA software V.16. ${ }^{13}$ There was no patient or public involvement in this project.

\section{RESULTS}

There were 42290 patients with newly recorded AF during the study. At diagnosis, 28\% (11 722)), 45\% (19 055) and 27\% (11 513) of patients were aged 65-74, 75-84 and 85+ years, respectively; $49 \%$ (20 850) of patients in the total sample were female. The proportion of new cases diagnosed in female patients rose from 39\% (4583) in the $65-74$ years age group to $61 \%$ (6994) in the over 85 years age group;. $35 \%$ (14861) of cases were diagnosed in those with four or more pre-existing comorbidities, falling to $6 \%$ (2492) in those with no other comorbidities with no systematic variation by deprivation. Across all age groups, there were falling numbers of cases in the later years of the study, this represents the falling number of practices contributing to CPRD using the Vision computer software over this time period (table 1).

Both cohort and period effects are seen in the prescription of anticoagulation over the 10 years of the study. For all age groups, the proportion of patients anticoagulated at the time of diagnosis increased between 2008 and 2018 (figure 2, table 2). This change is most marked in those aged $85+$ years, where there was a rise from $27 \%$ (679) in 2008-2010 to $74 \%$ (865) in 2016-2018. During the same time period, anticoagulation for the 65-74 years age group rose from the higher baseline of $55 \%$ (1482) to $86 \%$ (937), and for the $75-84$ years age group from $54 \%$ (2339) to $86 \%$ (1430). The difference in the proportion of those anticoagulated between the oldest and youngest age groups narrowed considerably over the 10-year period, from $28 \%$ to $11 \%$. Within each age and year of diagnosis cohort, there is also a consistent upward trend in the proportion who are anticoagulated over time. For example, in those aged 85+ years during 2008-2010 the proportion anticoagulated at diagnosis was $27 \%$ (679). By 2016-2018, of those who remained in this cohort, now aged $95+$ years, $55 \%$ (56) are receiving treatment.

No patients diagnosed with AF in 2008/2009 were prescribed a DOAC, but by $2017 / 2018,70 \%$ (1222) received a DOAC within 1 year of diagnosis (figure 3 ). DOACs represented $92 \%(1222 / 1319)$ of first anticoagulant prescriptions for AF in 2017/18. The use of DOACs increased rapidly in all age groups, with the largest increases seen between 2012 and 2016. The rate of increase was initially highest in the $85+$ years age group. There was no significant difference between age groups in the proportion given a DOAC from 2016 onwards (figure 3). 


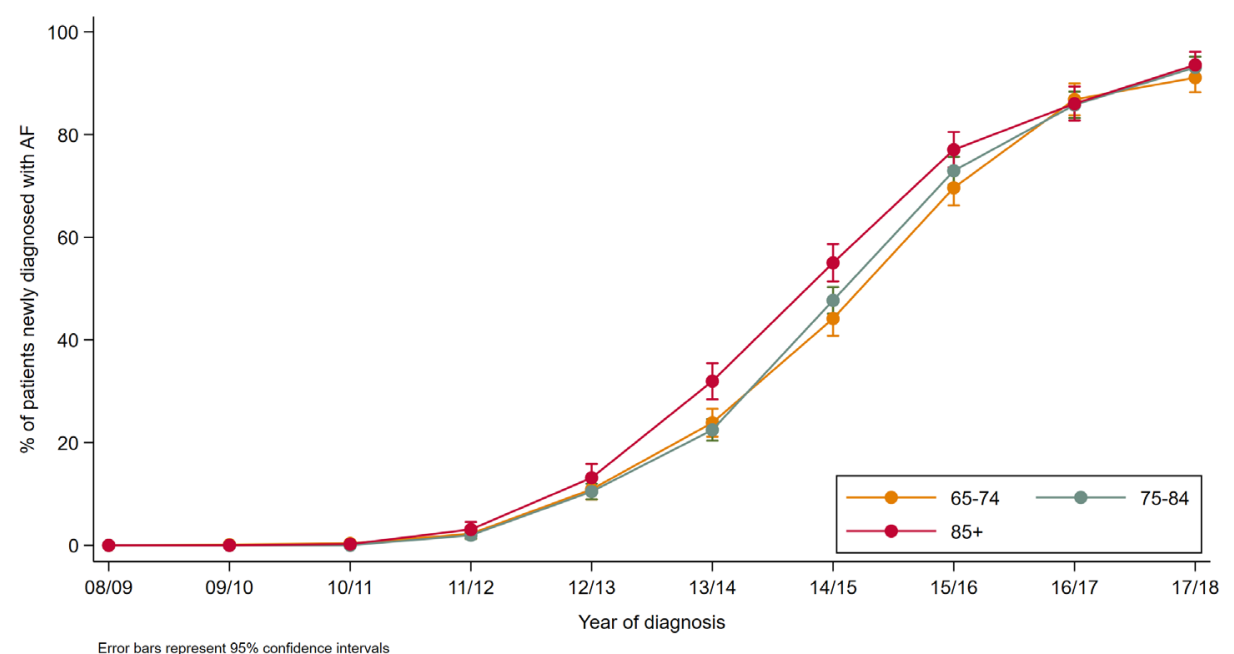

Figure 3 The proportion of first anticoagulation prescriptions for newly diagnosed atrial fibrillation (AF) that are for a direct oral anticoagulant.

\begin{tabular}{llllr}
\hline \multicolumn{7}{l}{ Table 3} & Type of anticoagulation by age & & \\
\hline Age at diagnosis & $\mathbf{6 5 - 7 4}$ & $\mathbf{7 5 - 8 4}$ & $\mathbf{8 5 +}$ & All ages \\
\hline DOAC only & 2379 & 3761 & 2211 & 8351 \\
Warfarin only & 5653 & 8831 & 3269 & 17753 \\
Warfarin changed to DOAC & 1125 & 1646 & 527 & 3298 \\
\hline DOAC changed to warfarin & 78 & 125 & 43 & 246 \\
\hline
\end{tabular}

A total of 29644 patients received a prescription for oral anticoagulation at any time point following AF diagnosis. Of these, $60 \%$ (17 753) received only warfarin, $28 \%$ (8351) only DOAC, $11 \%$ (3298) switched from warfarin to DOAC during the study period and $1 \%$ (246) changed from DOAC to warfarin. Due to the small number of patients who changed from DOAC to warfarin, these patients were excluded from further analysis (table 3 ).

Persistence with anticoagulation was high across all age groups, with the greatest rate of decrease in the first year (figure 4). Anticoagulation persistence was higher in the younger age groups at all time points. At 5 years, the survivor function for anticoagulation was $0.79(95 \%$ CI 0.78 to 0.81 ) in those aged $65-74$ years, 0.73 (95\% CI 0.72 to 0.75$)$ in those aged $75-84$ years and 0.58 (95\% CI 0.54 to 0.61 ) in those aged $85+$ years.

Of those who received a single type of anticoagulation, persistence was highest in those prescribed a DOAC (figure 5). At 5 years, the probability of continuing

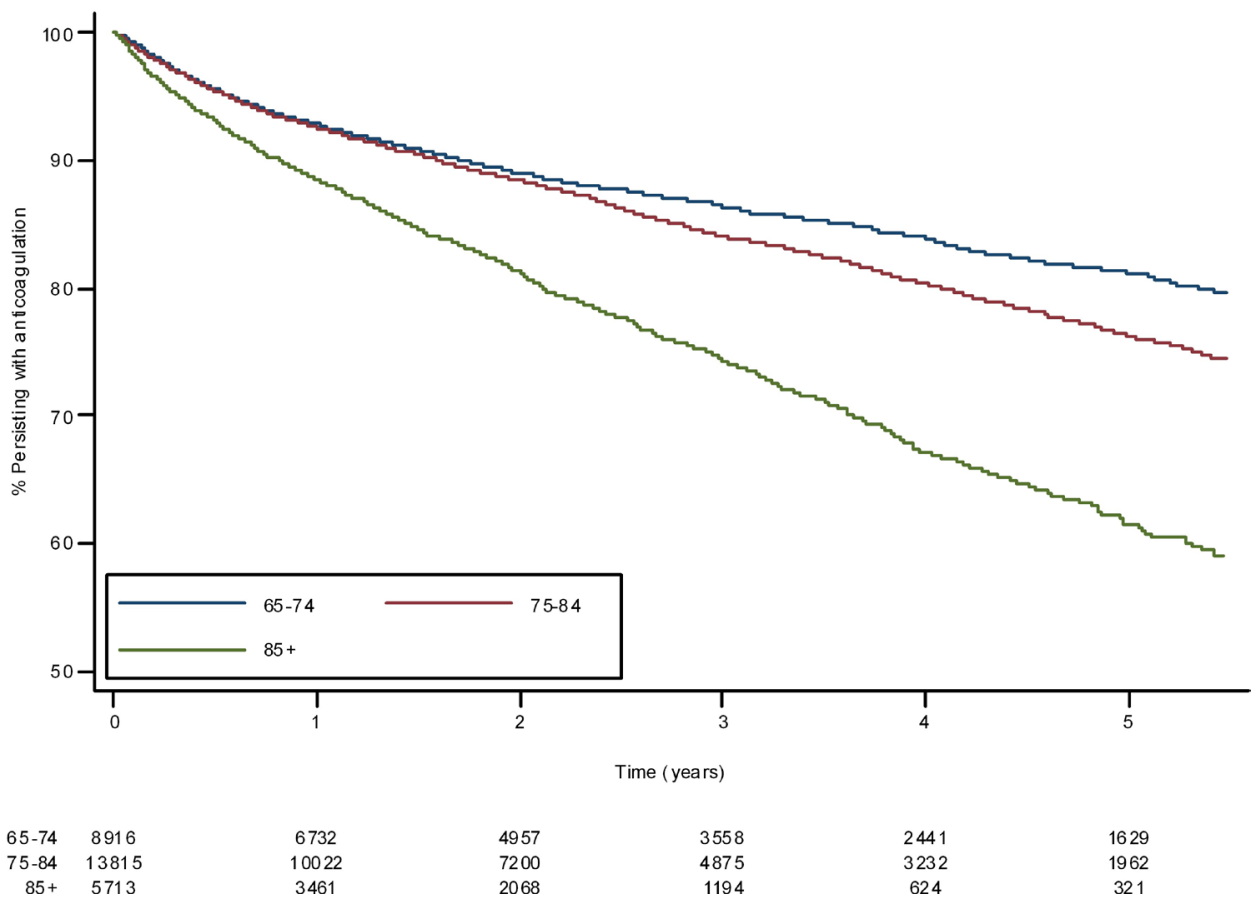

Figure 4 Anticoagulation persistence by age. 

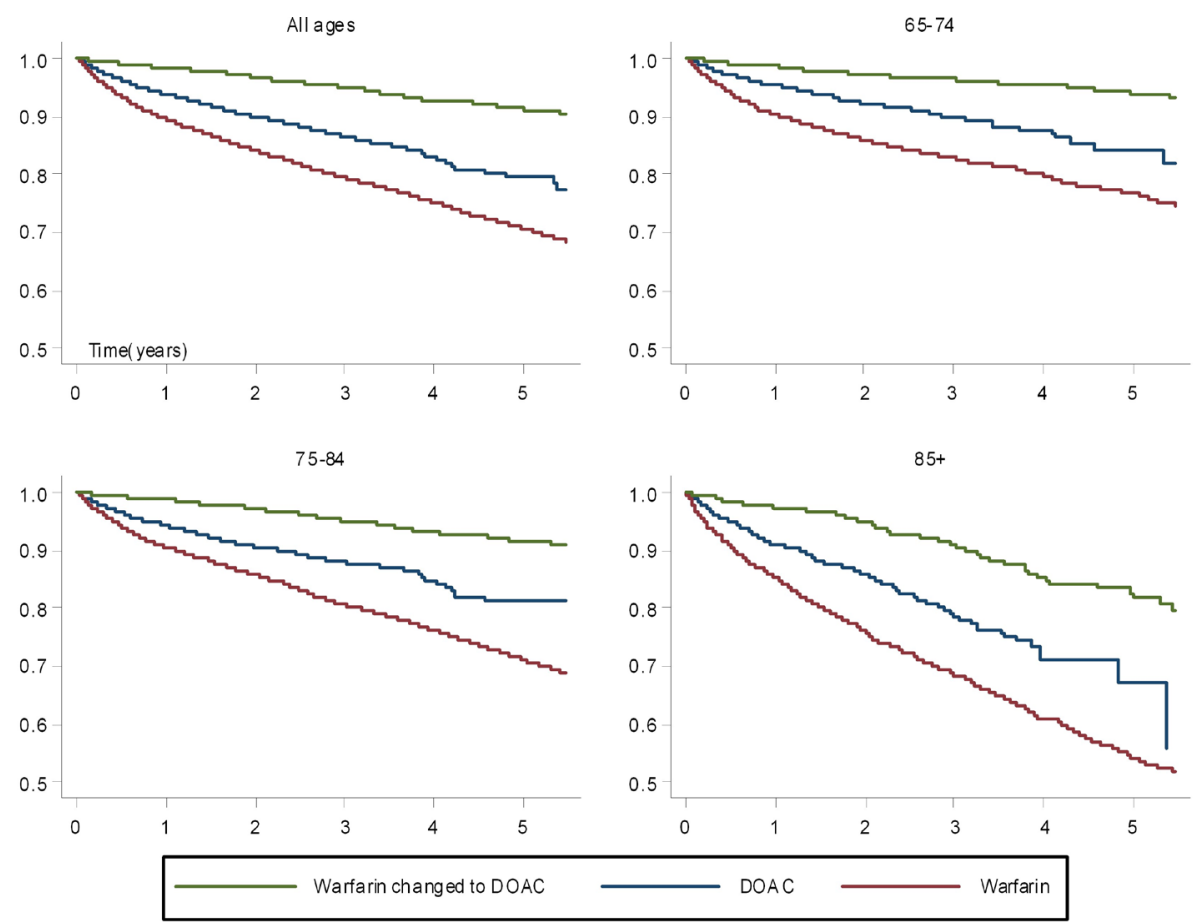

\begin{tabular}{|c|c|c|c|c|c|c|c|c|c|c|c|c|}
\hline & \multicolumn{3}{|c|}{ All ages } & \multicolumn{3}{|c|}{$65-74$} & \multicolumn{3}{|c|}{$75-84$} & \multicolumn{3}{|c|}{$85+$} \\
\hline Time(Years) & DOAC & Warfarin & $\begin{array}{l}\text { Warfarin } \\
\text { changed } \\
\text { to DOAC }\end{array}$ & DOAC & Warfarin & $\begin{array}{l}\text { Warfarin } \\
\text { changed } \\
\text { to DOAC }\end{array}$ & DOAC & Warfarin & $\begin{array}{l}\text { Warfarin } \\
\text { changed } \\
\text { to DOAC }\end{array}$ & DOAC & Warfarin & $\begin{array}{l}\text { Warfarin } \\
\text { changed } \\
\text { to DOAC }\end{array}$ \\
\hline 1 & 4786 & 12210 & 3021 & 1522 & 4082 & 1050 & 2197 & 6216 & 1520 & 1070 & 1912 & 453 \\
\hline 2 & 2531 & 8917 & 2650 & 851 & 3112 & 941 & 1185 & 4611 & 1343 & 495 & 1194 & 367 \\
\hline 3 & 1136 & 6233 & 2193 & 401 & 2324 & 798 & 536 & 3190 & 1120 & 200 & 719 & 277 \\
\hline 4 & 419 & 4149 & 1689 & 154 & 1634 & 636 & 205 & 2127 & 884 & 63 & 390 & 172 \\
\hline 5 & 128 & 2539 & 1226 & 52 & 1073 & 496 & 62 & 1264 & 629 & 14 & 206 & 102 \\
\hline
\end{tabular}

Figure 5 Anticoagulation persistence by age and type. Due to small patient numbers those who have transferred from direct oral anticoagulant (DOAC) to warfarin are not presented.

anticoagulation for those only prescribed a DOAC was 0.79 (95\% CI 0.77 to 0.82$)$ compared with $0.71(95 \%$ CI 0.71 to 0.76$)$ in those only prescribed warfarin. If all patients initially prescribed warfarin are considered, including those who subsequently switched to a DOAC, then 5-year probability of continuing anticoagulation was 0.75 (95\% CI 0.740.76).

\section{DISCUSSION}

\section{Summary}

Anticoagulation has increased across all age groups from 2008 to 2018 with the greatest rise in those aged over 85 years. There have been both cohort and period effects with regard to prescription of anticoagulation. Increases are greatest in those with newly diagnosed AF. DOACs now represent the vast majority of anticoagulant prescriptions offered to all patients aged over 65 years with newly diagnosed AF. Anticoagulation persistence decreases with age, being lowest in those aged over 85 years. Persistence is higher in those prescribed DOACs as compared with warfarin.

\section{Strengths and limitations}

The major strength of this study is the large sample size and generalisability to the UK population. Ours is the first study to tease out period from cohort effects in anticoagulation prescribing in this age group. As with all electronic record studies a major limitation is the reliance on recorded, coded, diagnoses. It is possible that patients receiving anticoagulation are more likely to have a coded diagnosis of $\mathrm{AF}$ and therefore the incidence of anticoagulation seen in this paper may be artificially elevated.

Inclusion of only patients who have had an influenza vaccination may mean that the cohort of patients described in this study are those more likely to attend for healthcare and therefore more likely to receive anticoagulation. However, the AF incidence seen is comparable to that reported using all CPRD records. ${ }^{14}$ These potential biases are unlikely to have had any major effect on the observed trends, since their magnitude will not have changed substantially over the time period of the study.

A further limitation is the use of anticoagulant prescription issues as a marker for persistence with medication. It is possible that prescriptions are issued without 
medication being taken, particularly considering the move toward electronic repeat dispensing in the UK.

We have shown that anticoagulation goes up over time in all age groups. This is likely in part due to increased use of anticoagulation but may also represent differential survival in those who are anticoagulated.

\section{Comparison with existing literature}

The trends in overall anticoagulation and the use of DOAC as the anticoagulant of choice we present are consistent with previous UK studies. ${ }^{415}$ Our work brings these results up to date and presents new data with regard to age stratification for those aged over 65 yers.

We show that people who were started on a DOAC tend to persist longer than those on warfarin; however, there may be both patient selection and time effects. DOACs were initiated in later years than warfarin and there has been, over the period of the study, increased knowledge and awareness of the importance of persisting with anticoagulation. What we have observed is similar to the persistence seen in a recent meta-analysis where pooled persistence with DOACs was higher than vitamin $\mathrm{K}$ antagonists (OR 1.44), ${ }^{16}$ although many of the studies included within this have a significantly shorter period of follow-up. Anticoagulation persistence is known to be lower in younger age groups, ${ }^{17} 18$ our patients were older, mean age 80 years, than in any of the studies included in the systematic review and this may partially explain the high overall persistence seen. In clinical trials where randomisation eliminates patient selection bias, there was no clear pattern of superiority of DOAC over warfarin. ${ }^{19-21}$ Our study is observational and therefore we cannot be sure of the effects of patient selection bias. The choice of anticoagulant is largely an individual decision made between clinician and patient and there may be an unmeasured characteristic that affects the choice of medication and the likelihood of persistence.

\section{Implications for research policy and practice}

Our results show there has been a major shift in the use of anticoagulation in the UK. DOACs have become the firstline anticoagulant for those with $\mathrm{AF}$ and are being used in ever increasing numbers of patients, including large numbers aged over 85 years. We provide some circumstantial evidence to support the success of initiatives to increase uptake of anticoagulation. ${ }^{22}$ This suggests that poor uptake of anticoagulation may no longer be a barrier to screening for AF.

We note that the high levels of anticoagulant prescribing that we observed are against a background of multimorbidity, which would be expected to increase the potential iatrogenic effects of anticoagulation prescribing, as multimorbidity increases risk of haemorrhage. ${ }^{23}$

Those prescribed warfarin require frequent monitoring and dose adjustment. Currently, there are multiple models of care as to how this service is provided, across both primary and secondary care. As the number of patients receiving warfarin continues to decline, consideration will need to be given to the best model of care for continuation of specialist warfarin services for the small number of patients who continue to use it. This will need to account for the likely older age profile of these patients.

A final consideration is that the current data are all drawn prior to the COVID-19 pandemic. As a result of the pandemic, UK guidance, endorsed by the Royal College of General Practitioners, has encouraged the accelerated switching of warfarin to DOAC for patients with AF to avoid unnecessary trips to healthcare settings for monitoring. ${ }^{24}$ Pandemic guidance from NHS England explicitly recommends that patients newly diagnosed with $\mathrm{AF}$ are offered a DOAC in preference to warfarin. Therefore, the trends seen in this paper may well have accelerated in 2020 .

\section{CONCLUSION}

The use of anticoagulation for AF has increased over the period 2008-2018, with the greatest increases seen in those aged over 85 years, both in terms of initiation and persistence than previously reported. DOACs now represent the vast majority of anticoagulation prescriptions for newly diagnosed AF. This study provides some evidence that DOACs are associated with higher long-term persistence in older adults than warfarin.

Twitter Jenny Lund @J_C_Lund

Contributors $\mathrm{JL}$ wrote the protocol and statistical analysis plan, cleaned and analysed the data, drafted and revised the paper. CLS wrote the statistical analysis plan, drafted and revised the paper. DE wrote the protocol and revised the draft paper. JM wrote the protocol and revised the draft paper.

Funding JL's work for this study is supported by the Wellcome Trust as part of the Wellcome Trust PhD Programme for Primary Care Clinicians (grant number RHZB/195). CLS received salary support from the National Institute for Health Research (NIHR) School for Primary Care Research (project reference SPCR-201410043 Capacity Building Award 10a SPCR Postdoctoral Fellowship Award). JM is an NIHR Senior Investigator.

Disclaimer The views expressed are those of the author(s) and not necessarily those of the Wellcome Trust, NIHR or the Department of Health and Social Care.

Competing interests None declared.

Patient consent for publication Not required.

Ethics approval Permission for the CPRD to receive and supply anonymous patient data for generic public health research is granted directly to the CPRD by the National Research Ethics Service of the UK Health Research Authority. Regulatory approvals to use CPRD data for the current project were granted by the CPRD Independent Scientific Advisory Committee (ISAC protocol 17_141RMn).

Provenance and peer review Not commissioned; externally peer reviewed.

Data availability statement Data may be obtained from a third party and are not publicly available. Deidentified data used for this study are available from CPRD (https://cprd.com/Data-access). Access to CPRD data is subject to protocol approval by an Independent Scientific Advisory Committee (ISAC). ISAC is a nonstatutory expert advisory body established in 2006 by the Secretary of State for Health to provide scientific advice on research requests to access data provided by CPRD.

Open access This is an open access article distributed in accordance with the Creative Commons Attribution 4.0 Unported (CC BY 4.0) license, which permits others to copy, redistribute, remix, transform and build upon this work for any purpose, provided the original work is properly cited, a link to the licence is given, and indication of whether changes were made. See: https://creativecommons.org/ licenses/by/4.0/. 
ORCID iD

Jenny Lund http://orcid.org/0000-0001-7727-9925

\section{REFERENCES}

1 Wolf PA, Abbott RD, Kannel WB. Atrial fibrillation: a major contributor to stroke in the elderly. The Framingham study. Arch Intern Med 1987;147:1561-4.

2 Lane DA, Wolff A, Shantsila E, et al. Optimising stroke prevention in patients with atrial fibrillation. Br J Gen Pract 2015;65:117.

3 Robson J, Homer K, Ahmed Z, et al. Variation in anticoagulation for atrial fibrillation between English clinical commissioning groups: an observational study. Br J Gen Pract 2018;68:e551-8.

4 Loo SY, Dell'Aniello S, Huiart L, et al. Trends in the prescription of novel oral anticoagulants in UK primary care. Br J Clin Pharmacol 2017;83:2096-106.

5 Torn M, Bollen WLEM, van der Meer FJM, et al. Risks of oral anticoagulant therapy with increasing age. Arch Intern Med 2005;165:1527-32.

6 Zathar Z, Karunatilleke A, Fawzy AM, et al. Atrial fibrillation in older people: concepts and controversies. Front Med 2019;6:175.

7 Criteria for appraising the viability, effectiveness and appropriateness of a screening programme: public health England, 2015. Available: https://www.gov.uk/government/publications/evidence-reviewcriteria-national-screening-programmes/criteria-for-appraisingthe-viability-effectiveness-and-appropriateness-of-a-screeningprogramme [Accessed 5 Oct 2020].

8 King S, Fitzgerald A, Bartlett C, Mahon J, Arber M, Carr E, Glanville J. Evidence summary for screening for atrial fibrillation in adults. external review against programme appraisal criteria for the UK national screening Committee UK National Screening Committee; 2019.

9 Herrett E, Gallagher AM, Bhaskaran K, et al. Data resource profile: clinical practice research datalink (CPRD). Int J Epidemiol 2015;44:827-36.

10 Adult flu vaccination coverage: Nuffield trust, 2020. Available: https://www.nuffieldtrust.org.uk/resource/adult-flu-vaccinationcoverage [Accessed 17 Nov 2020].

11 Payne RA, Mendonca SC, Elliott MN, et al. Development and validation of the Cambridge multimorbidity score. CMAJ 2020;192:E107-14.

12 English indices of deprivation 2019: Ministry of housing, communities and local government, 2019. Available: https://www. gov.uk/government/statistics/english-indices-of-deprivation-2019 [Accessed 25 May 2021].

13 StataCorp. Stata Statistical Software:Release 16. College Station, TX: StataCorp LLC, 2019.

14 Mendonça SC, Saunders CL, Lund J, et al. Temporal trends in incidence of atrial fibrillation in primary care records: a populationbased cohort study. BMJ Open 2020;10:e042518.

15 Adderley N, Ryan R, Marshall T. The role of contraindications in prescribing anticoagulants to patients with atrial fibrillation: a crosssectional analysis of primary care data in the UK. Br J Gen Pract 2017;67:e588-97.

16 Ozaki AF, Choi AS, Le QT, et al. Real-World adherence and persistence to direct oral anticoagulants in patients with atrial fibrillation: a systematic review and meta-analysis. Circ Cardiovasc Qual Outcomes 2020;13:e005969.

17 Banerjee A, Benedetto V, Gichuru P, et al. Adherence and persistence to direct oral anticoagulants in atrial fibrillation: a population-based study. Heart 2020;106:119-26.

18 Zielinski GD, van Rein N, Teichert M, et al. Persistence of oral anticoagulant treatment for atrial fibrillation in the Netherlands: a surveillance study. Res Pract Thromb Haemost 2020;4:141-53.

19 Granger CB, Alexander JH, McMurray JJV, et al. Apixaban versus warfarin in patients with atrial fibrillation. $N$ Engl J Med 2011;365:981-92.

20 Patel MR, Mahaffey KW, Garg J, et al. Rivaroxaban versus warfarin in nonvalvular atrial fibrillation. N Engl J Med 2011;365:883-91.

21 Connolly SJ, Ezekowitz MD, Yusuf S, et al. Dabigatran versus warfarin in patients with atrial fibrillation. $N$ Engl J Med 2009;361:1139-51.

22 TheAHSNNetwork. Atrial fibrillation. detect, protect, perfect, 2019.

23 Proietti M, Marzona I, Vannini T, et al. Long-Term relationship between atrial fibrillation, multimorbidity and oral anticoagulant drug use. Mayo Clin Proc 2019;94:2427-36.

24 Williams H. Guidance for the safe switching of warfarin to direct oral anticoagulants (DOACs) for patients with non-valvular AF and venous thromboembolism (DVT / PE) during the coronavirus pandemic: Royal pharmaceutical Society, 2020. Available: https:// www.rpharms.com/Portals/0/RPS\%20document\%20library/Open\% 20access/Coronavirus/FINAL\%20Guidance\%20on\%20safe\% 20switching \%20 of\%20warfarin\%20to\%20DOAC\%20COVID-19\% 20Mar\%202020.pdf? ver=2020-03-26-180945-627 [Accessed 6 Jul 2020]. 